\title{
Diffusive and percolative lattice migration: Excitons ${ }^{a)}$
}

\author{
Raoul Kopelman and Panos Argyrakis \\ Department of Chemistry, The University of Michigan, Ann Arbor, Michigan 48109 \\ (Received 19 September 1979; accepted 19 November 1979)
}

\begin{abstract}
A microscopic transport theory is developed for stochastic and correlated hopping on ordered and random lattices that contain a small fraction of supertraps and a small number of "hoppers" (i.e., excitons). It includes short-time ("transient") behavior, which is of interest for both time-resolved and steady-state experiments. The relations with diffusion, percolation, random walk, and rate equations are exhibited and applications to energy transport in disordered molecular aggregates illustrate the approach, which is a combination of a rigorous analytical method and simple computer simulations of general validity. Simple analytical results, derived for special (limiting) cases, are compared with other methods, thus emphasizing the roles of time, dimensionality, anisotropy, clusterization, correlation of hops, and the order parameter of the lattice as well as the suitability of various approaches for dealing with these factors.
\end{abstract}

\section{INTRODUCTION}

The concept and equations of diffusion in a continuous medium have been formulated by Einstein ${ }^{1}$ and Smoluchowski. ${ }^{2}$ Einstein emphasized that the simple equations, as well as the concept of a "diffusion constant," break down at very short times and, equivalently, over very small domains of space. In addition, the random ("Brownian") motion is usually assumed to oc cur in a homogeneous space. Considering that both spatial and temporal resolution have been dramatically improved in recent years, the above restrictions are of increasing relevance. In addition, in certain experiments, like energy transport, even simple techniques yield results that depend on short time behavior (i.e., due to the short lifetime of an excitation) or small domain behavior (i.e., due to the microscopic inhomogeneity of the material under investigation). The drastic effects due to the inhomogeneity of the medium were pointed out twenty years ago by Frish and Hammersley, ${ }^{3}$ who suggested that under such conditions the concept of diffusion should be replaced by that of "percolation" or "random percolation." The latter term implies a random walk on a random lattice, and "random lattice" implies that the lattice sites (or bonds) are either "open" or "closed" at random (with a definite probability) to the motion of the (random) walker. The most striking difference between diffusive and percolative motion occurs below a certain critical value of the ori " parameter describing the random medium (i.e., a critical concentration of open lattice sites). This critical point is defined by the connectivity ("topology," "coordination number") of the lattice, which in turn is practically defined by the effective range of a migration "step."

There is now renewed interest in this topic, which has been mainly spurted by the current interest in energy transport through disordered systems, be they inorganic crystals like ruby, ${ }^{4}$ inorganic glasses like europium phosphate glass, ${ }^{5}$ isotopic mixed organic crystals like naphthalene, ${ }^{6}$ or photosynthetic systems in chloroplasts. ${ }^{7,8}$ Also, as the analytical formulation of such problems encounters formidable difficulties, it

\footnotetext{
a) Supported by NIH Grant 2R01 NS08116-10A 1 .
}

is now prudent to circumvent these by the appropriate use of computer simulation. 90

Interesting recent work on this problem includes that by Haan and Zwanzig, ${ }^{11}$ who discussed a "generalized diffusion" that is both time and space dependent. They discussed excitation migration on a random lattice with a small concentration of impurity sites (from which the excitation is excluded) and showed that over short periods the migration is "nondiffusive," while for longer times it appeared to be "diffusive" under a certain set of simplifying assumptions. Alexander et al. ${ }^{12}$ derived equations for "percolative" motion on a disordered linear chain. Ching and Huber ${ }^{9}$ have investigated the validity of some "ad hoc" analytical expressions for energy transport on disordered lattices by comparison with computer simulations, and Huber recently applied a coherent potential approximation to the master equa tions. ${ }^{13}$ It was pointed out some time ago ${ }^{14}$ that there is a severe difficulty in deriving rigorous and closed analytical solutions for the analogous problem of energy states and bands. The most sophisticated approaches, such as Green's function, average $t$-matrix method, coherent potential approximations, and moment expansions have not led to satisfactory solutions in the intermediate concentration range, where cluster structure and percolation are of importance. ${ }^{15}$ This is the reason why problems of energy localization and "absence of diffusion" (Anderson localization) are currently being investigated by numerical methods. ${ }^{16}$ On the other hand, detailed classical or quantum mechanical simulations of donor-donor and donor-acceptor transfer would be too expensive, both in terms of computer time and that of computer storage space. A solution we have suggested earlier involves an appropriate "mix" of analytical formulae and Monte Carlo simulations. ${ }^{10}$ We now continue this effort, using results of simulations to show some conditions under which conventional diffusion and/or Stern-Volmer rate equations are justified. We also give an approach to the general problem, which is valid for short and intermediate time random walks on lattices, for correlated walks on lattices, and for random and correlated walks on random lattices.

Our approach starts from a directly measurable quantity, the sensor registration probability $P(t)$. This probability is related to time dependent rate and dif- 
fusion "constants," which in certain limits become true constants. Our examples include simulations on binary random lattices and their applications to the energy migration on ternary lattices containing sites of hosts, guests (donors), and sensors (acceptors, "supertraps"). Results for binary and simple ("perfect") lattices are also given and compared with previous approaches. We point out that our basic approach is that of a modified hopping model. Very recently, the hopping model ${ }^{13,17}$ has been shown to apply when the donor-donor transfer is about equal to or greater than the donor-sensor transfer rate. This is the physical case of interest to us here.

In Sec. II we define the transfer probability and relate it to variables of our model (such as migration ef ficiency). In Sec. III we set up a standard rate equation, in which we incorporate a time-dependent rate constant. In Sec. IV we discuss the Stern-Volmer limit for different degrees of coherence of motion and show how this can be treated by simulation techniques. The efficiency of random walk motion is related to known exact results for one-dimensional (linear), two-dimensional (square), and three-dimensional (simple cubic) lattices. In Sec. $V$ we discuss the diffusion model and show that it applies only as a limiting case of our more general model. In Sec. VI we give a simple limiting result relating the migration efficiency and the coordination number for correlated walk transfer in a perfect lattice. In Sec. VII we discuss the distinction between diffusion and percolation, and in Sec. VIII we provide some numerical illustrations for the solutions of the rate equations in microscopically heterogeneous systems. These are based on our computer simulations. The example is the naphthalene singlet exciton system.

\section{REGISTRATION PROBABILITY AND MIGRATION EFFICIENCY}

In a binary lattice with random substitutional dis order, we define a quasilattice of energy carrying sites (guests) with total concentration (mole fraction) $C$, where

$$
0<C<1 \text {. }
$$

A small random fraction $S$ of these sites are supertraps (total absorbers):

$$
0<S \ll 1 \text {. }
$$

We attribute to the supertrapping process an efficiency $\gamma$ (usually of the order of unity), defined by the fraction of "visits" to the supertrap that result in absorption (the absorption is always total, i.e., the probability of back transfer from the supertrap is zero). We define by $n(t)$ the average number of distinct sites visited at least once after time $t$ (in the absence of traps). We also define the probability of supertrapping ("registration," "capture"), after time $t$, as $P(t)$, where

$$
0<P(t)<1 \text {. }
$$

$n(t)$ can also define the "spread" of the excitation. In a connected lattice $n(t)$ and $P(t)$ increase monotonically with time. We note that $n(t)$ depends also on the lattice topology, the concentration $C$, and the details of the mi- gration (stochastic or correlated, range of transfer, etc.). An elementary argument gives

$$
\begin{aligned}
& 1-P=(1-S \gamma)^{n}, \\
& 1-P=e^{-S m}, \quad \gamma S \ll 1, \\
& P(t)=1-e^{-\operatorname{Srn}(t)} .
\end{aligned}
$$

We emphasize that the above equations are for an "average" excitation in a connected lattice or quasilattice. If isolated microscopic domains or minicluster $s^{6,18}$ exist, a modified treatment gives, for substitutionally random lattices,

$$
P(t)=P_{\infty}\left(1-e^{-S m(t)}\right)+S I_{2 \mathrm{z}},
$$

where $P_{\infty}$ is the percolation probability of the carrier quasilattice and $I_{2 v}$ is the average minicluster size. ${ }^{6,19}$ Here we assumed that the migration inside the miniclusters is essentially instantaneous or, alternatively, that the excitation is effectively delocalized throughout the minicluster. We note that well above the critical concentration one has

$$
\begin{aligned}
& P_{\infty} \rightarrow 1, \quad C \gg C_{c}, \\
& I_{2 \nabla} \rightarrow 0, \quad C \gg C_{c},
\end{aligned}
$$

giving again Eq. (4c) instead of Eq. (4d).

We define a dimensionless time $\theta$, such that the average hopping time $t_{h}$ is given by

$$
t_{\mathrm{h}}=t / \theta \text {. }
$$

$\theta$ is thus also an average number of "hops" ("steps"). In a perfect lattice with nearest neighbor interactions $\theta$ reduces to the number of hops or the number of steps (sites visited, counting each visit separately). We now define $e^{20}$ the migration efficiency $\epsilon$ as

$$
\epsilon=n / \theta \text {, }
$$

and also the hopping rate (time independent),

$$
\beta=t_{h}^{-1}
$$

giving instead of $\mathrm{Eq} .(4 \mathrm{c})$,

$$
P(t)=1-e^{-a \epsilon(t) t},
$$

where

$$
a=S \gamma \beta
$$

is a time independent constant. We also get, similar to Eq. (4d),

$$
P(t)=P_{\infty}\left(1-e^{-a \epsilon(t) t}\right)+S I_{2 x}
$$

\section{MIGRATION RATE EQUATIONS}

We assume that the population of walkers (i.e., excitation energy) decays via two independent channels: (1) the above-mentioned registration (supertrapping, capture) process, and (2), an exponential decay, i.e., due to luminescence and additional nonradiative processes. The second process has an associated lifetime $\tau$ and a simple decay rate

$$
k=\tau^{-1} \text {. }
$$

We define the fraction of sites occupied by walkers (i.e., 
properly excited carriers) as $\rho$ and assume henceforth that

$$
\rho \ll 1 .
$$

If $\rho=\rho_{0}$ at $t=0$, then it follows that

$$
\rho(t)=\rho_{0}[1-P(t)] e^{-k t} \text {. }
$$

We get therefrom

$$
\partial \ln \rho / \partial t=-k-(1-P)^{-1}(\theta P / \partial t) .
$$

Defining a migration Konstant (spelled this way to emphasize its time dependence)

$$
K(t) \equiv(1-P)^{-1}(\partial P / \partial t),
$$

one gets the familiar so-called "rate equation":

$$
d \rho / d t=-k \rho-K(t) \rho,
$$

which turns into a conventional rate equation

$$
d \rho / d t=-(k+K) \rho
$$

only in the Stern-Volmer limit, ${ }^{21}$ where

$$
K \neq f(t) \text {. }
$$

The generalized migration Konstant $K(t)$ can be easily related to the migration efficiency. Using Eqs. (9) and (16) one gets

$$
K(t)=a[\epsilon+t(\partial \epsilon / \partial t)], \quad C \gg c .
$$

which shows that the Stern-Volmer limit of Eq. (19) is achieved in this case only for

$$
\epsilon \neq f(t)
$$

giving

$$
K=a \epsilon .
$$

The more general result for $K(t)$, based on $\mathrm{Eq}$. (11), is

$$
K(t)=\frac{P_{\infty} a e^{-a \epsilon t}[\epsilon+t(d \epsilon / d t)]}{1-P_{\infty}\left(1-e^{-a t t}\right)-S I_{\mathrm{av}}} .
$$

This shows that for a disordered lattice with $P_{\infty} \neq 1$ one does not get the Stern-Volmer limit even for $\epsilon \neq f(t)$.

Equivalent but slightly more general results for $K(t)$ can be given in terms of $n(t)$, the number of distinct sites visited by time $t$. From Eqs. (4c) and (16) one gets for a binary lattice $(C=1)$ or for $C \gg c$.

$$
K(t)=S \gamma(B n / \partial t) \text {, }
$$

while for a general ternary lattice one gets from Eqs. (4d) and (16)

$$
K(t)=\frac{P_{\infty} S \gamma e^{-S m}(\partial n / \partial t)}{1-P_{\infty}\left(1-e^{-S m}\right)-S I_{a v}} .
$$

\section{EXAMPLES AND THE STERN-VOLMER LIMIT}

\section{A. "Coherent" motion and self-avoiding walk on a perfect lattice}

Assume that the excitation moves in a straight line, that is, it has perfect directional memory. Thus, after each of the $\theta$ steps a new site has been visited. Obviously $\epsilon=1$ and $K=a$, giving a Stern-Volmer kinetics. Note that this result is true for any dimensionality. It is also true for the much more general case of self - avoiding walk where also always $\epsilon=1$. In these cases the meaning of $a$ [Eqs. (9), (10)] is that of an "average hopping rate" from a guest to an effective supertrap.

\section{B. Simple random walk on perfect cubic lattices}

Montroll ${ }^{22}$ has shown that for a simple cubic lattice, the asymptotic solution $(t \rightarrow \infty)$ gives

$$
n=0.629462 \theta \text {, }
$$

and thus

$$
\epsilon=0.629462 \text {, }
$$

giving again the Stern-Volmer result. However, an improved formula, using an "asymptotic expansion," gives for cubic lattices

$$
\epsilon=A+B \theta^{-1 / 2}+C \theta^{-3 / 2}+\cdots,
$$

where $A, B$, and $C$ are constants (i.e., $A=0.629462$ for a simple cubic lattice). This indicates that for finite times $\epsilon$ is a function of time, and so is $K$ :

$$
K=a A+a B \theta^{-1 / 2}+\cdots \text {. }
$$

\section{Simple random walk on a square lattice}

Here the asymptotic formula ${ }^{22}$ gives

$$
\epsilon=\pi / \log \theta=f(t),
$$

which is time dependent even for very long times (with a limiting value of zero). Thus, even in this simplest random walk on a two-dimensional lattice, $K=f(t)$ and the Stern-Volmer limit [Eq. (19)] does not hold (except for the trivial case of $\theta \rightarrow \infty$, where it vanishes " $\log$ arithmically").

\section{Simple random walk on a linear chain}

The limiting behavior ${ }^{22}$ for $t-\infty$ is

$$
\epsilon=(8 / \pi)^{1 / 2} \theta^{-1 / 2},
$$

which is time dependent, with additional terms to be added $^{22}$ at shorter times (involving $\theta^{-3 / 2}, \theta^{-5 / 2}$, etc.). Again, the Stern-Volmer limit is not approached asymptotically (and again $K \rightarrow 0$ as $t \rightarrow \infty$ ).

\section{E. Results based on Monte Carlo calculations}

Simple random walk (i.e., nearest neighbor steps only) results, for short times, on perfect lattices of various dimensions, are easily obtained by Monte Carlo simulations [Figs. 1(a), 1(b)]. These simulations are particularly useful for “correlated" random walks, i.e., where the walker has partial directional memory. If the walker has perfect memory for $l$ steps and then loses memory completely, one gets the same result as for a simple random walk on a superlattice (with a supercell of length $l$ times the unit-cell length). Counting, however, "time" on the single step scale, it becomes obvious that the asymptotic limit is approached " $l$ times slower." Thus, for a given timescale, this form of " $l$ walk" appears to be more sensitive to time than the simple random walk. In our simulations we let the "correlation length" vary, randomly, around a mean value $l$, with a standard deviation $d$. As long as $d$ is significantly 

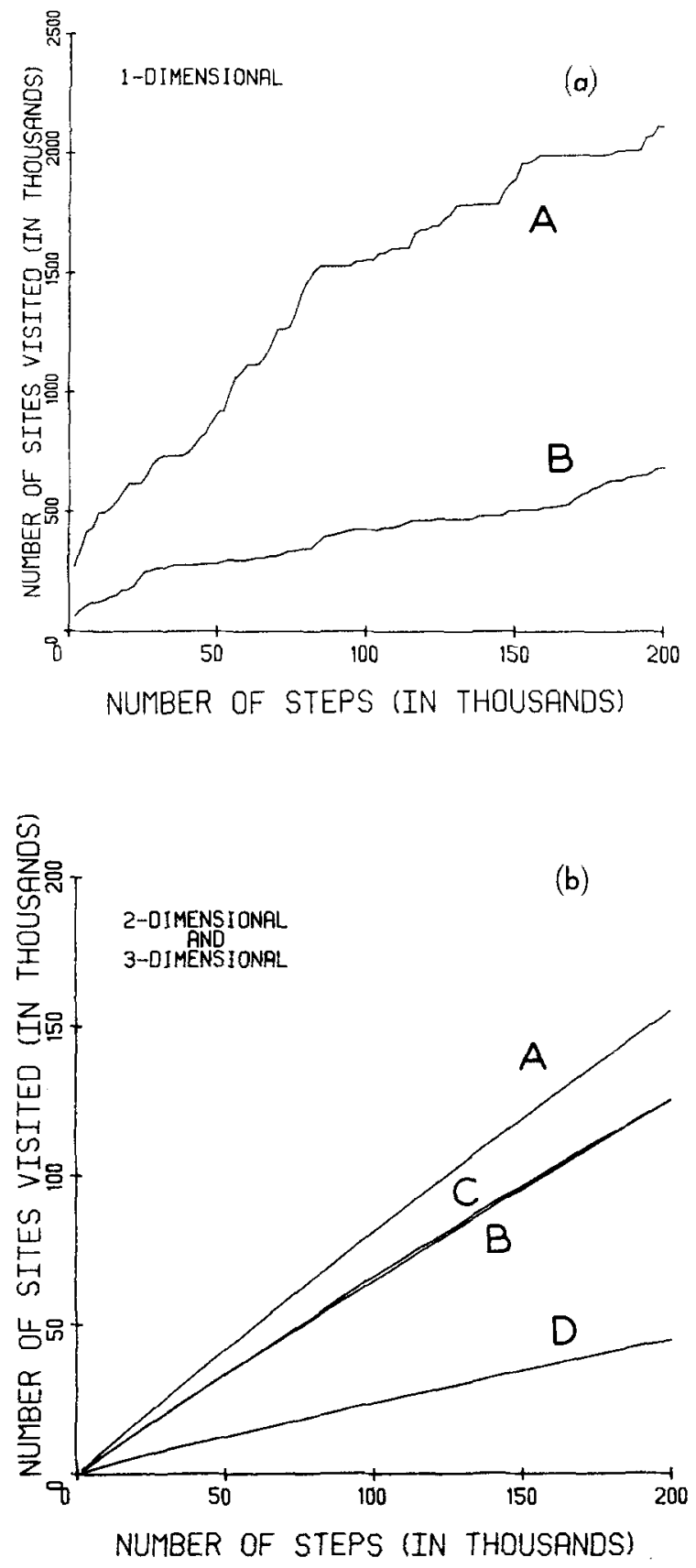

FIG. 1. Plot of the number of distinct sites visited at least once by a random walker as a function of the total number of steps. Part (a) shows the one-dimensional behavior for correlation values of $l=1$ (curve $B$ ) and $l=10$ (curve $A$ ) (Gaussian distribution with a standard deviation $d$ equal to 3.0 ). Part (b) includes two-dimensional and three-dimensional examples for the same correlation values. In all cases a one component crystal is employed. For the two-dimensional case, four interactions of equal magnitude were used on a $1022 \times 1022$ lattice, while for the three-dimensional case the lattice was simple cubic $(101 \times 101 \times 101)$ with six nearest neighbor jumps. The correlation values are as follows: three-dimensional $l=10$, $d=3.0$ : curve $A ; l=1, d=0$ : curve $B$; two-dimensional $l=10$, $d=3.0$ : curve $C ; l=1, d=0$, curve $D$. Note the similarity between curve $B(3-d)$ and $C(2-d)$.

smaller than $l$, the qualitative behavior is still that of a correlated walk with a fixed $l$ value. More on correlated walks is given in Sec. VI.

\section{DIFFUSION, RANDOM WALK, AND DIMENSIONALITY}

Powell and Soos ${ }^{23}$ have used a "transfer rate" $k_{D}(t)$ which is equivalent to our rate coefficient $K(t)$. While their coefficient is nominally related to excitation dif fusion in an anisotropic medium, their expression is apparently derived from a random walk formalism based on an asymptotic formula for the isotropic cubic lattice that is equivalent to our equation (26) with only the first two terms retained. We suggest the following "translation" for their $D R_{A}$ ("diffusion constant" times "activator radius"):

$$
D R_{A}=(4 \pi)^{-1} V_{m} \beta \gamma \bar{\epsilon},
$$

where $V_{m}$ is a molecular volume, as also defined by Powell and Soos. ${ }^{23}$ Here $\bar{\epsilon}$ is the limiting value for the isotropic cubic lattice, i.e., 0.629 for the simple cubic lattice, as given by Montroll. ${ }^{22}$

We emphasize the following restrictions on this equiv alence between the Powell-Soos model and ours:

1. Long time behavior $(t-\infty)$;

2. Isotropic cubic lattice;

3. Simple random walk, that is, with only nearest neighbor steps $(t=1)$;

4. No energy barrier (host) sites $(C=1)$.

Under "realistic" conditions, as in our own naphthalene experiments, ${ }^{24-26}$ none of the se restrictions is justifiable. The exciton transfer probability is very anisotropic and closer to being effectively two dimensional. ${ }^{6,27}$ Also, the short-time energy transfer dominates both the timeresolved and the steady-state investigations. In addition, the hops are most probably correlated at low tem-

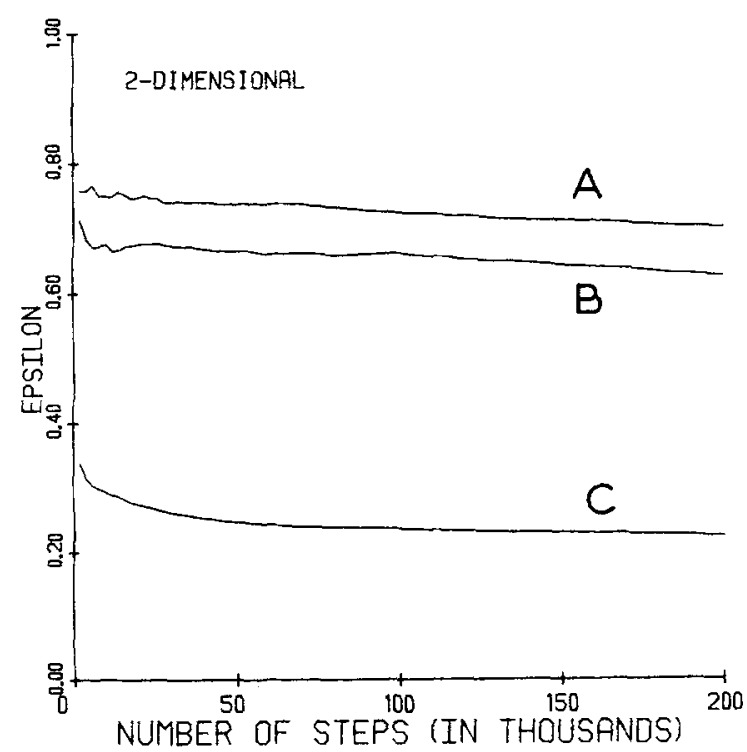

FIG. 2. Visitation efficiency $\epsilon$ as a function of the number of steps. The definition is $\epsilon=n / \theta$, and this plot is derived from the results of Fig. 1. Here three cases are shown for a square two-dimensional lattice. Correlation values of $l=1,10$, and 100 (with the standard deviation $d$ of the distribution equal to 0 , 3.0 , and 30.0, respectively) result in curves $C, B$, and $A$, respectively. 


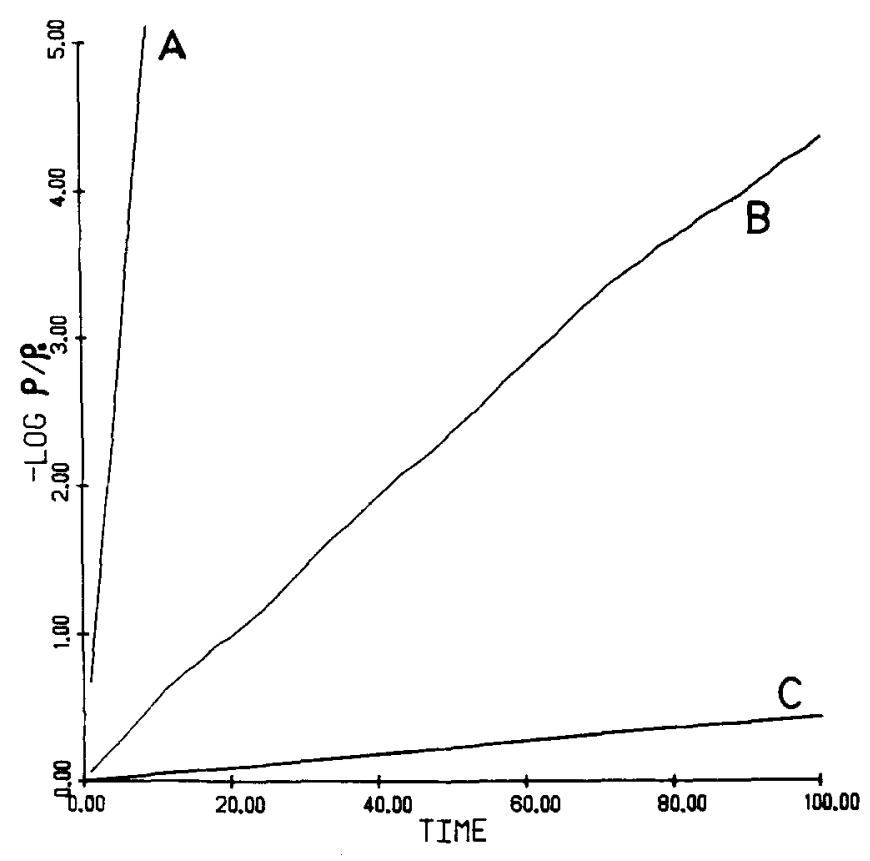

FIG. 3. A plot of $-\log \left(\rho / \rho_{0}\right)$ as a function of time. Here the result of Eq. (34) is plotted for three different supertrap concentrations, $10^{-5}$ (curve $C$ ), $10^{-4}$ (curve $B$ ), and $10^{-3}$ (curve $A$ ) mole fraction, for a random walk on a $500 \times 500$ two-dimensional surface, and represents the hypothetical limiting case of an infinite exciton lifetime. Here we have plotted time explicitly, using the relation 1 time unit $=2000$ actual steps. For the derivation of the solution, see text. Notice that the solutions are not really exponential because $n(t)$ is not a linear function of time.

peratures (i.e., $l \gg 1$ ) and many of the experiments are for energy carrier concentrations of 0.6-0.9 (see below). To emphasize how long a time it takes to reach "diffusive behavior," compared to the exciton lifetime $\left(10^{-7} \mathrm{sec}\right)$ or the time resolution of the experiments $\left(10^{-9}\right.$ sec), we show a few examples of the migration efficiency $\epsilon$ versus time derived from Monte Carlo simulations (Fig. 2). Other diffusion formalism approaches (e.g., Heber $\left.^{28}\right)$, with results very similar to those of Powell and Soos, ${ }^{23}$ are based on dubious boundary conditions, as has been pointed out by Haan and Zwanzig, ${ }^{11}$ in addition to the basic flaws mentioned above: (1) Assumption of isotropic three-dimensional diffusion; (2) Assumption of times long enough and distances large enough to justify a diffusion constant; (3) A perfect superlattice of energy carriers, rather than a random quasilattice. (4) A macroscopic-sized supertrap volume, amenable to a diffusion approach. We notice that the "conventional" diffusion approach ignores the effects of the local struc ture (lattice versus continuum) and the short time ("transient") effects.

The conventional approach to diffusion has one important advantage compared with our approach: It does provide information on the space-time evolution of the excitation while our approach at this stage is limited to obtaining information only about the time evolution of the exciton migration. We foresee a solution, however, to the combined space-time problem in terms of simulation studies, studies that relate the excitation "spread" $n(t)$ to an actual excitation volume.
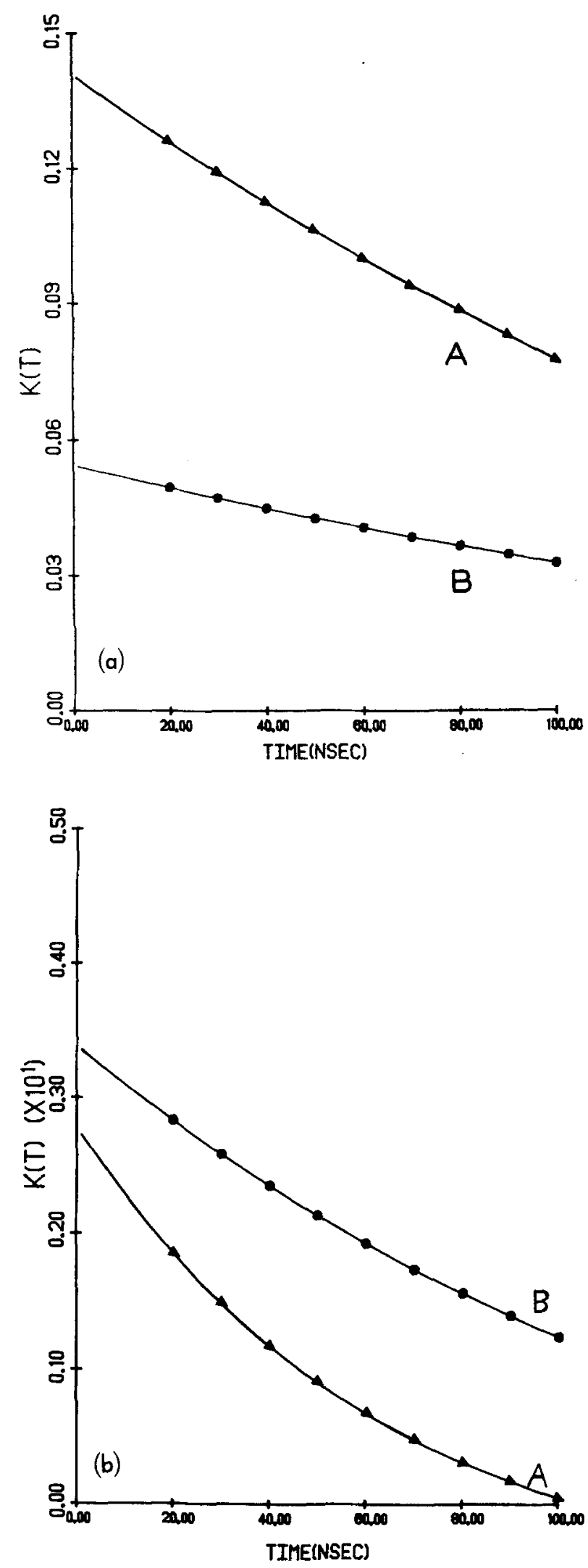

FIG. 4. Time-dependent rate constant behavior. The time dependent rate constant $K(t)$ is plotted as a function of time, according to Eq. (20). The solution is given for the case of a pure crystal (a) and a case of a mixed crystal (b) made of $70 \%$ "open" and $30 \%$ "closed" sites, for two correlation values, $l=1$ (curves $B$ ) and $l=10$ (with $d=3.0$ ) (curves $A$ ). The units of $K(t)$ are effective supertrap sites visited/nsec. The lattice sizes are $500 \times 500$. 

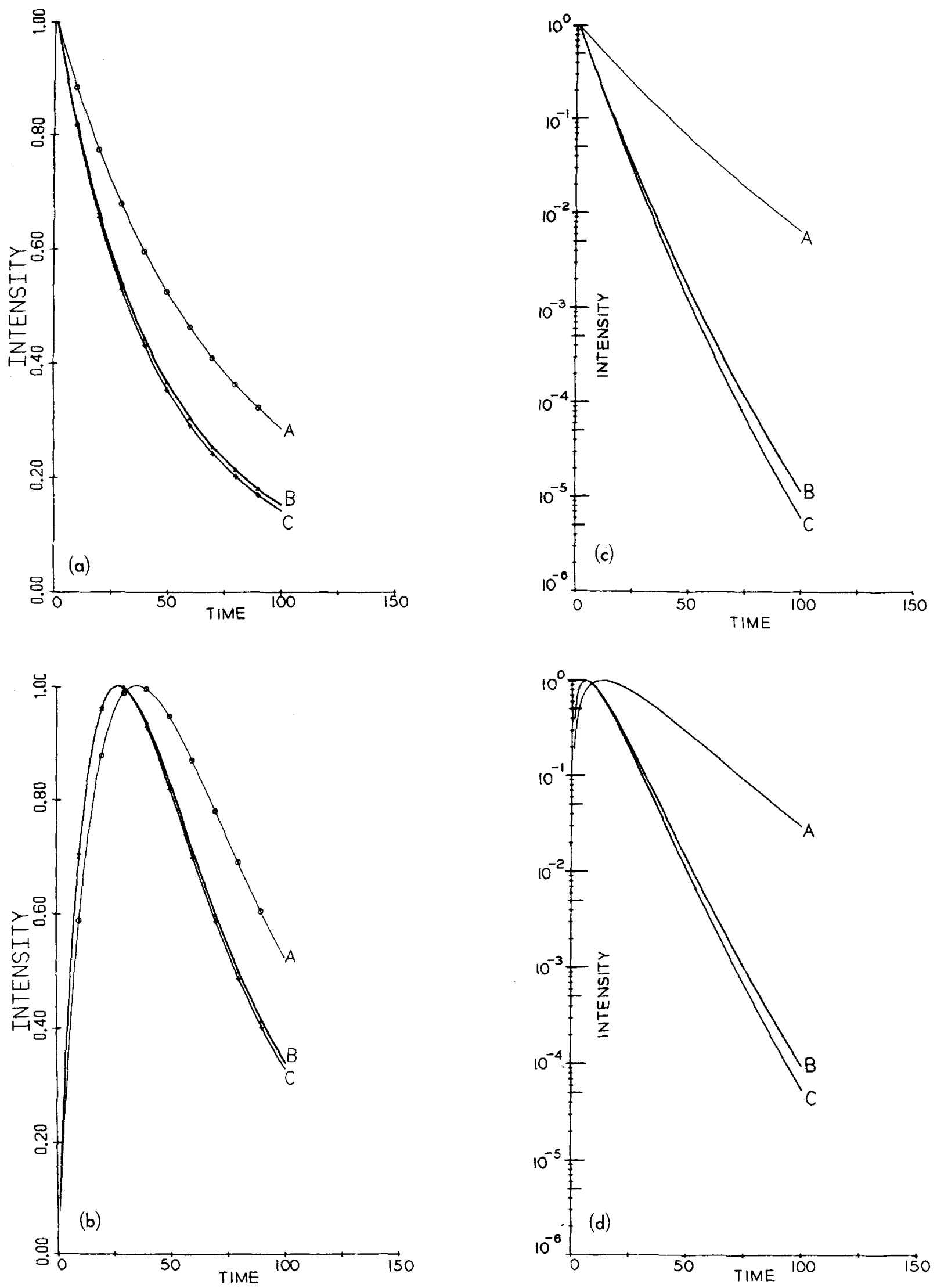

FIG. 5. Complete solution of $\rho$ as a function of time. Plot $a$ is for the naphthalene exciton decay while plot $b$ is that for the BMN. plots $c$ and $d$ are simply the logarithmie plots of $a$ and $b$. This figure is similar to Fig. 3 but with the inclusion of the time-independent term in the rate equation. The solution is derived elsewhere (Ref. 26) and the plot is given here only for illustrative purposes. Here a two-dimensional $(500 \times 500)$ square topology is considered and the three cases of $l=1$, 10 , and 100 are shown (with standard deviation for the distribution equal to $0,3.0$, and 30.0 , respectively) by curves $A, B$, and $C$, respectively. 
We note here that Powell and Soos ${ }^{23}$ have also used a GRW (generalized random walk) model, where, in principle, they allowed for an anisotropic trapping region and for variable random steps. However, these parameters were not really utilized in comparison to actual experiments. Moreover, the main drawbacks remain: (1) the use of an asymptotic behavior $(t \rightarrow \infty)$, and (2) limitation to a three-dimensional (isotropic) walk. Thus in our limit of isotropic random walk at long time [Eq. $\left.\left(24^{\prime}\right)\right]$, we can make the following connection. The trapping capacity $C(A)$ of Powell and $\operatorname{Soos}^{23}$ is

$$
C(A)=\epsilon \gamma, \quad \epsilon \neq f(t) .
$$

\section{CORRELATED HOPS ON A LATTICE, KINETICS AND DIFFUSION}

For the simple case, where each "random step" simply consists of $l$ lattice units, it is obvious that the simple random walk formulas (nearest neighbor steps on a lattice) only need a trivial alteration. Also, in the limit of long time (large $\theta$ ), the motion again becomes diffusive. Once there is a "distribution of hops" the result is less trivial.

We give here a simple result for a special case of correlated random hops, ${ }^{10}$ for which

$$
\vec{N} \gg \theta \gg l \gg d \gg 1 \text {. }
$$

Here $\vec{N}$ is the size of the lattice (number of sites), $\theta$ the number of steps, $l$ the average correlation number (number of correlated steps), and $d$ its standard deviation (assuming a Gaussian distribution around $l$ ). For lattices of dimension higher than unity one has

$$
\epsilon \equiv \frac{n}{\theta} \sim \frac{b-2}{b-1} \text { [for Eq. (31) conditions] }
$$

where $b$ is the coordination number (bond order). Thus, for $t-\infty$ (and $\bar{N}-\infty$, where $\bar{N} \gg \theta$ ), the efficiency is time independent and thus one has Stern-Volmer kinetics, which is consistent with a diffusive behavior.

\section{RANDOM LATTICE MIGRATION, DIFFUSION, AND PERCOLATION}

For random walk on a random lattice it is not at all clear whether for $t \rightarrow \infty$ one gets conventional diffusion. ${ }^{11,29,30}$ We actually believe that a diffusive limit does not exist in general. This can be demonstrated by examples. For instance, for nearest-neighbor-only hops it is obvious that below the critical percolation concentration in a random binary lattice (made of "sites" and "vacancies") no conventional diffusion can occur. The intuitive reason lies in the recognition that the random walker is confined in a given "minicluster." This case has also been discussed in the literature for exponentially decreasing hopping probabilities. ${ }^{31,32}$ We can say, following Hammersley, ${ }^{3}$ that in disordered lattices the concept of diffusion is actually replaced by the concept of percolation. Only in special cases can we expect the percolative migration to result in conventional dif fusion.

\section{NUMERICAL ILLUSTRATIONS}

If we assume an infinite "natural" lifetime $\tau$, then $k=0$ and Eq. (14) simplifies. In Fig. 3, we give a plot of $\log \left(\rho / \rho_{0}\right)$ versus $t$ for $k=0$. From Eqs. (4c) and (14) we see that for a one-component crystal

$$
\begin{gathered}
\rho(t)=p_{0} e^{-S m(t)} e^{-k t} \\
\text { or, for } k=\tau^{-1}=0, \\
-\log \left[\rho(t) / \rho_{0}\right]=S \gamma n(t)=a \epsilon(t) t .
\end{gathered}
$$

This simple result immediately indicates that we do not expect a linear dependence of $\log \rho(t)$ with $t$ because of the time dependence of $\epsilon$. The extent of deviation from linearity (i.e., deviation from exponential decay) is shown in Fig. 3 for various values of $S \gamma$. Incidentally, the fast rise with time of $\log \left(\rho / \rho_{0}\right)$ for $S=10^{-3}$ justifies the inclusion of this supertrap concentration case in the limit of "supertransfer."19,27

We have given in Fig. 2 some numerical illustrations of the migration efficiency $\epsilon$ as a function of time (or number of steps). We show here a few examples of the rate Konstant $K(t)$ describing supertrapping in an ordinary crystal (containing just small amounts of supertraps), and in a substitutionally random binary crystal containing (in addition to the supertraps) a large mole fraction of vacancies, i.e., sites inaccessible to the walker (i.e., exciton). Figure 4 shows this for both simple and correlated random walks.

We also show illustrations of the "walking exciton" population $\rho$ (see above), as a function of time [Figs. $5(\mathrm{a})$ and $5(\mathrm{~b})]$, as well as the complementary population of supertrapped (nonwalking) excitons. The two quantities can be directly compared with the typical time-resolved experimental information, ${ }^{25,28,33}$ namely, the luminescence intensities of the free and trapped exciton emissions (which are differentiated by their spectral locations, i.e., the energy of the emitted light quanta). Many further illustrations will be given in conjunction with experiments in a forthcoming paper. ${ }^{33}$

${ }^{1} \mathrm{~A}$. Einstein, Investigations on the Theory of the Brownian Movement (Dutton, New York, 1926).

${ }^{2}$ M. V. Smoluchowski, Phys. Z. 17, 557, 585 (1916).

${ }^{3}$ H. L. Frish and J. M. Hammersley, J. Soc. Indust. Appl. Math: 11, 894 (1963).

${ }^{4}$ P. M. Selzer, D. L. Huber, B. B. Barnett, and W. M. Yen, Phys. Rev. B 17, 4979 (1978).

${ }^{5}$ M. J. Weber, Phys. Rev. B 4, 2932 (1971).

${ }^{6} \mathrm{R}$. Kopelman, in Radiationless Processes in Molecules and Condensed Phases, edited by F. K. Fong, Topics in Applied Physics, Vol. 15 (Springer, Berlin, 1976).

${ }^{7}$ A. J. Campillo and S. L. Shapiro, Photochem. Photobiol. 28, 975 (1978).

${ }^{8}$ C. E. Swenberg, N. E. Geacintov, and J. Breton, Photochem Photobiol. 28, 999 (1978).

${ }^{9}$ W. Y. Ching and D. L. Huber, Phys. Rev. B 18, 5320 (1978).

${ }^{10} \mathrm{P}$. A rgyrakis and R. Kopelman, J. Theor. Biol. 73, 205 (1978).

${ }^{11}$ S. W. Haan and R. Zwanzig, J. Chem. Phys. 68, 1879 (1978); S. W. Haan, Ph. D. thesis, University of Maryland, 1977.

${ }^{12} \mathrm{~S}$. Alexander, J. Bernasconi, and R. Orbach, Phys. Rev. B 17, 4311 (1978).

${ }^{13}$ D. L. Huber, Phys. Rev. B (in press). 
${ }^{14} \mathrm{H}-\mathrm{K}$. Hong and R. Kopelman, J. Chem. Phys. 55, 5380 (1971).

${ }^{15}$ R. Kopelman, in Excited States $I I$, edited by E. C. Lim (Academic, New York, 1975), p. 33.

${ }^{16} \mathrm{D}$. J. Thouless, in Les Houches-Condensed Phase, edited by R. Balian et al. (North-Holland, Amsterdam, 1979), p. 1.

${ }^{17} \mathrm{D}$. L. Huber, in Laser Spectroscopy of Ions and Molecules in Solids, edited by W. M. Yen and P. M. Selzer (Springer,

Berlin, to be published).

${ }^{18}$ J. Hoshen, R. Kopelman, and E. M. Monberg, J. Stat. Phys. 19,219 (1978).

${ }^{19} \mathrm{~J}$. Hoshen and R. Kopelman, J. Chem. Phys. 65, 2817 (1976).

${ }^{20}$ R. Kopelman, Mol. Cryst. Symp. VIIIth, Santa Barbara, CA, (1977), p. 174.

${ }^{21} \mathrm{~J}$. B. Birks, Organic Molecular Photophysics, Vol. 2 (WileyInterscience, New York, 1975).

${ }^{22}$ E. W. Montroll, J. Math. Phys. 10, 753 (1969).
${ }^{23}$ R. C. Powell and Z. G. Soos, J. Lumin. 11, 1 (1975).

${ }^{24}$ P. Argyrakis and R. Kopelman, J. Chem. Phys. 66, 3301 (1977).

${ }^{25}$ P. Argyrakis and R. Kopelman, Chem. Phys. Lett. 61, 187 (1979).

${ }^{26} \mathrm{P}$. A rgyrakis, Ph. D. thesis, University of Michigan, 1979.

${ }^{27}$ R. Kopelman, E. M. Monberg, and F. W. Ochs, Chem. Phys. 21, 373 (1977).

${ }^{28}$ J. Heber, Phys. Status Solidi B 48, 319 (1971).

${ }^{29}$ K. Godzik and J. Jortner, Chem. Phys. Lett. 63, 3 (1979); Chem. Phys. 38, 277 (1979).

${ }^{30} \mathrm{G}$. R. Gochanour, H. C. Anderson, and M. D. Fayer, J. Chem. Phys. 70, 4254 (1979).

${ }^{31}$ N. F. Mott and E. A. Davis, Electronic Processes in NonCrystalline Materials (Oxford U. P., New York, 1978), 2nd ed.

${ }^{32}$ M. Pollak and M. L. Knotek, J. Non-Cryst. Solids 32, 141 (1979).

${ }^{33}$ P. Argyrakis and R. Kopelman (unpublished). 